\title{
Apoptosis of HL-60 human leukemia cells induced by Asiatic acid through modulation of B-cell lymphoma 2 family proteins and the mitogen-activated protein kinase signaling pathway
}

\author{
QIULING WU ${ }^{1 *}$, TINGTING LV ${ }^{1,2^{*}}$, YAN CHEN $^{1}$, LU WEN $^{1}$, JUNLI ZHANG $^{1}$, \\ XUDONG JIANG ${ }^{1}$ and FANG LIU ${ }^{1}$
}

\author{
${ }^{1}$ Institute of Hematology, Union Hospital, Tongji Medical College, Huazhong University of Science and Technology, \\ Wuhan, Hubei 430022; ${ }^{2}$ Department of Hematology, Xiangyang Center Hospital, Xiangyang, Hubei 441021, P.R. China
}

Received June 11, 2014; Accepted February 20, 2015

DOI: $10.3892 / \mathrm{mmr} .2015 .3534$

\begin{abstract}
The toxicities of conventional chemotherapeutic agents to normal cells restrict their dosage and clinical efficacy in acute leukemia; therefore, it is important to develop novel chemotherapeutics, including natural products, which selectively target cancer-specific pathways. The present study aimed to explore the effect of the chemopreventive agent asiatic acid (AA) on the proliferation and apoptotic rate of the leukemia cell line HL-60 and investigated the mechanisms underlying its anti-tumor activity. The effect of AA on the proliferation of HL-60 cells was evaluated using the MTT assay. Annexin V-fluorescein isothiocyanate/propidium iodide double staining followed by flow cytometric analysis as well as Hoechst 33258 staining were used to analyze the apoptotic rate of the cells. Furthermore, changes of survivin, B-cell lymphoma 2 (Bcl-2), myeloid cell leukemia 1 (Mcl-1), extracellular signal-regulated kinase (ERK), c-Jun N-terminal kinase (JNK) and p38 expressions were detected by western blot analysis. AA blocked the growth of HL-60 cells in a dose- and time-dependent manner. The $\mathrm{IC}_{50}$-value of $\mathrm{AA}$ on HL-60 cells was $46.67 \pm 5.08 \mu \mathrm{mol} / 1$ for $24 \mathrm{~h}$. AA induced apoptosis in a dose-dependent manner, which was inhibited in the presence of Z-DEVD-FMK, a specific inhibitor of caspase. The anti-apoptotic proteins $\mathrm{Bcl}-2, \mathrm{Mcl}-1$ and survivin were downregulated by AA in a dose-dependent manner. Concurrently, AA inhibited ERK and p38 phosphorylation in a dose-dependent manner, while JNK phosphorylation was
\end{abstract}

Correspondence to: Professor Fang Liu, Institute of Hematology, Union Hospital, Tongji Medical College, Huazhong University of Science and Technology, 1277 Jiefang Street, Wuhan, Hubei 430022, P.R. China

E-mail: liufang1@medmail.com.cn

*Contributed equally

Key words: asiatic acid, leukemia, apoptosis, b-cell lymphoma 2, mitogen-activated protein kinase not affected. In conclusion, the present study indicated that the p38 and ERK pathways, as well as modulation of Bcl-2 family and survivin proteins were key regulators of apoptosis induced in HL-60 cells in response to AA.

\section{Introduction}

Acute myeloid leukemia (AML) is an aggressive hematopoietic malignancy and primarily treated by chemotherapy. Although various chemotherapeutic agents have been developed for AML treatment, they affect normal cells, which causes unpleasant side effects, including hepatotoxicity, cardiotoxicity, hematotoxicity and infection, and which restricts their dosage and clinical efficacy. Moreover, resistance to conventional chemotherapeutic agents is a common reason for unsuccessful chemotherapy. Recent studies have suggested natural products as potent chemotherapeutic drugs for AML to improve the therapeutic efficacy and lower the side effects (1-3). For instance, $\beta$-elemene, an active component of the medicinal herb Curcuma wenyujin, induced apoptosis in human leukemia HL-60, NB4, K562 and HP100-1 cells through downregulation of cellular FLICE-like inhibitory protein and generation of reactive oxygen species (4).

In recent years, a series of pentacyclic triterpenoid compounds, including ursolic acid (5), oleanolic acid (6) and betulinic acid (7), were reported to exhibit anti-tumor activity, and therefore, triterpene acids are thought to have great potential as novel drugs for the treatment of malignant tumors. Asiatic acid (AA) is a pentacyclic triterpenoid derived from the medicinal plant Centella asiatica (family, Apiaceae). A wide range of beneficial effects of AA have been reported in hepatofibrosis (8), diabetes (9), ultraviolet (UV) radiation-induced photo aging and cerebral ischemia (10), and wound healing (11). Furthermore, AA has been reported to be cytotoxic to several solid tumor cell lines, including malignant glioma (12), human hepatoma (13), colon cancer (14), melanoma (15) and gastric cancer (16). Moreover, AA has attracted attention for its multiple protective effects against drug-induced hepatotoxicity, neurotoxicity and ulcers. Due to its potent anti-inflammatory, anti-cancer and chemo-protective activities, AA has been suggested to be a promising chemo- 
preventive and therapeutic agent. However, to the best of our knowledge, the effect of AA on hematological malignant cells has not been investigated to date. In the present study, the effect of AA on HL-60 cells was investigated using MTT cell proliferation assays and assessment of the apoptotic rate using flow cytometric analysis and confocal microscopy. Furthermore, the mechanism of the anti-cancer effect of AA was investigated by assessing changes in levels of B-cell lymphoma 2 (Bcl-2) family proteins and proteins involved in the mitogen-activated protein kinase (MAPK) signaling pathway using western blot analysis. The present study provided a molecular basis for the clinical application of AA in patients with acute leukemia.

\section{Materials and methods}

Materials. AA $\left(\mathrm{C}_{30} \mathrm{H}_{48} \mathrm{O}_{5}\right.$; molecular weight, $\left.488.7 \mathrm{~g} / \mathrm{mol}\right)$, dimethyl sulfoxide (DMSO), Hoechst 33258 and MTT were purchased from Sigma-Aldrich (St Louis, MO, USA). The Annexin V-fluorescein isothiocyanate (FITC)/propidium iodide (PI) reagent kit was purchased from Nanjing Key-Gen Biotech Co, Ltd (Nanjing, China). The bicinchoninic acid (BCA) Protein Assay kit, chemiluminescence reagent kit and polyvinylidene difluoride (PVDF) membranes were purchased from Pierce Biotechnology, Inc, USA. The rabbit polyclonal anti-extracellular signal-regulated kinase (ERK)1/2 (cat. no. 9102), rabbit monoclonal anti-phosphorylated (p-)ERK1/2 (cat. no. 4377), rabbit polyclonal anti-c-Jun N-terminal kinase (JNK) (cat. no. 9252), rabbit polyclonal anti-P-JNK (cat. no. 9251), rabbit polyclonal anti-p38 (cat. no. 9212), rabbit monoclonal anti-P-p38 (cat. no. 9215), rabbit polyclonal anti-Bcl-2 (cat. no. 2872), rabbit polyclonal anti-survivin (cat. no. 2803), rabbit polyclonal anti-myeloid cell leukemia 1 (Mcl-1) (cat. no. 4572), and mouse monoclonal anti- $\beta$-actin (cat. no. 3700) antibodies were provided by Cell Signaling Technology, Inc, (Danvers, MA, USA). Horseradish peroxidase-conjugated goat anti-rabbit (cat. no. 111-035-003) and donkey anti-mouse (cat. no. 715-035-150) immunoglobulin $\mathrm{G}$ secondary antibodies were purchased from Jackson Immuno Research Laboratories, Inc (West Grove, PA, USA). Z-DEVD-FMK was purchased from Medical and Biological Laboratories Co, Ltd, (Nagoya, Japan). Fetal bovine serum (FBS) was obtained from Hangzhou Sijiqing Biological Engineering Materials Co, Ltd (Hangzhou, China).

Cell culture. The leukemia cell line HL-60 was obtained from American Type Culture Collection (Rockville, MD, USA). Human peripheral blood mononuclear cells (PBMCs) from healthy volunteers (five males and five females between 20 and 50 years old) were obtained by Ficoll-Hypaque (Lonza Ltd., Wakersville, MD, USA) density gradient centrifugation at $1,000 \mathrm{xg}$, according to the manufacturer's instructions. All cells were cultured in RPMI 1640 medium (Invitrogen Life Technologies, Carlsbad, CA, USA) supplemented with $10 \%$ fetal bovine serum (FBS) and 1\% penicillin-streptomycin solution (Invitrogen Life Technologies) in a 5\% $\mathrm{CO}_{2}$ humidified atmosphere at $37^{\circ} \mathrm{C}$. Written informed consent for blood utilization was obtained from all volunteers. The procedures were approved by the ethics committee of Tongji Medical College, Huazhong University of Science and Technology (Hubei, China).

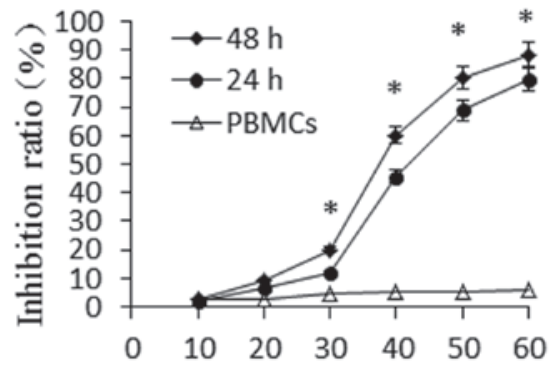

Concentration of AA $(\mu \mathrm{mol} / \mathrm{l})$

Figure 1. Analysis of the AA-induced proliferation inhibition ratio in HL-60 cells and human PBMCs. The cells were treated with various concentrations of AA for 24 and $48 \mathrm{~h}$. PBMCs were treated with AA for $24 \mathrm{~h}$. An MTT assay was performed to detect the proliferation inhibition ratio. Values are expressed as the mean \pm standard deviation of three independent experiments. ${ }^{*} \mathrm{P}<0.05$, as compared with the control cells in both 24 and $48 \mathrm{~h}$ treated cells. AA, asiatic acid; PBMCs, peripheral blood monocular cells.

Cell proliferation assay. The effects of AA on the proliferation of HL-60 cells and PBMCs were detected using an MTT assay. Briefly, the cells were maintained in RPMI-1640 medium (Thermo Fisher Scientific, Waltham, MA, USA) until reaching the mid-logarithmic growth phase and then seeded in 96-well plates with or without AA at various concentrations $(10,20,30,40,50$ and $60 \mu \mathrm{mol} / \mathrm{l})$ at a density of $2 \times 10^{5}$ cells per well. Following incubation for a set period of time, $20 \mu \mathrm{l}$ MTT $(5 \mathrm{mg} / \mathrm{ml})$ was added and the cells were incubated for another $3 \mathrm{~h}$ at $37^{\circ} \mathrm{C}$. The supernatant was discarded of and $150 \mu \mathrm{l}$ DMSO was added. The plate was gently agitated until the blue formazan crystals were fully dissolved. The absorbance (A) was measured at $490 \mathrm{~nm}$ using a microplate reader (Infinite F50; Tecan Spectra, Switzerland), and the cell proliferation inhibition ratio (\%) was calculated using the following formula: $\left[1-\left(\mathrm{A}_{\text {experimental sample }} / \mathrm{A}_{\text {control sample }}\right)\right] \times 100$.

Annexin V-FITC/PI double-labeled flow cytometry. The apoptotic rate was measured in HL-60 cells treated with AA at different concentrations alone or in combination with Z-DEVD-FMK. Four-color flow cytometry (FCM) was applied to detect the expression of Annexin V-FITC and the exclusion of PI. The cells positive for Annexin V-FITC and negative for PI represented the early apoptotic cells, whereas the cells positive for both markers represented the late apoptotic cells. The total apoptotic rate was the sum of the number of early and late apoptotic cells. Briefly, HL-60 cells were collected after the treatment using eppendorf tubes, washed twice with phosphate-buffered saline (PBS; Sigma-Aldrich) and resuspended in $500 \mu \mathrm{l}$ binding buffer. A total of $5 \mu \mathrm{l}$ Annexin V-FITC was added and the samples were maintained at room temperature for $10 \mathrm{~min}$. Next, $5 \mu \mathrm{l}$ PI was added and the cells were incubated for another $10 \mathrm{~min}$ in the dark. The fluorescence intensity was detected using a flow cytometer (Becton-Dickinson, Franklin Lakes, NJ, USA).

Hoechst 33258 staining. HL-60 cells were treated with $40 \mu \mathrm{mol} / 1 \mathrm{AA}$ for $24 \mathrm{~h}$ and the nuclear fragmentation was visualized using Hoechst 33258 staining. Briefly, $1 \times 10^{5}$ cells were seeded in 12-well plates and incubated with AA. After $24 \mathrm{~h}$, the cells were collected, washed twice with PBS and 


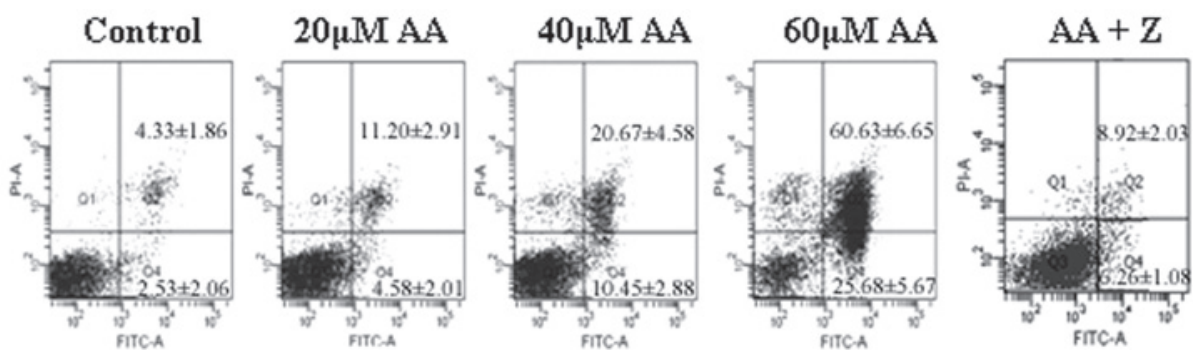

Figure 2. Assessment of the apoptotic rate of HL-60 cells induced by asiatic acid in various concentrations for $24 \mathrm{~h}$. Annexin V-FITC/PI double staining was applied followed by flow cytometric analysis. Q2 quadrant and Q4 quadrant represent late and early apoptotic cells, respectively. The total apoptotic rate was the sum of these two. FITC, fluorescein isothiocyanate; PI, propidium iodide.
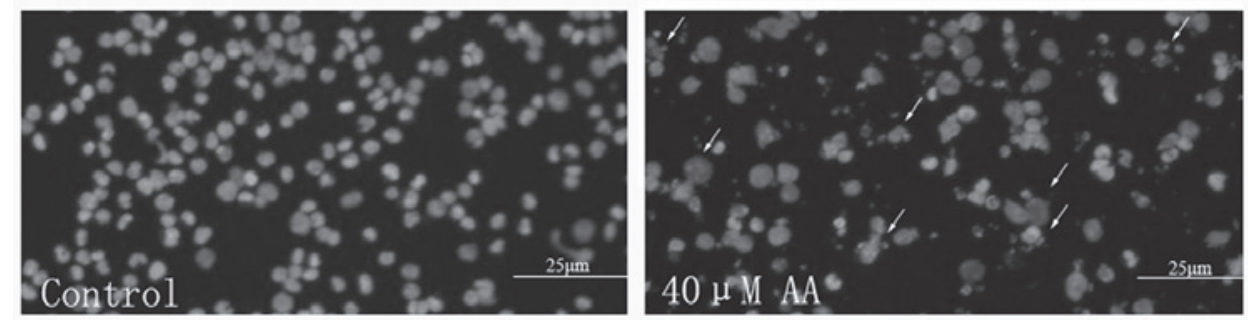

Figure 3. Effect of AA on the apoptosis of HL-60 cells. Following incubation with $40 \mu \mathrm{mol} / 1 \mathrm{AA}$ for $24 \mathrm{~h}$, the cells were stained with Hoechst 33258 and visualized using a fluorescence microscope. The number of apoptotic bodies exhibiting fragmented or condensed nuclei (indicated by the arrows) were detected in the AA-treated group but not in the control group. AA, asiatic acid.

fixed in $4 \%$ paraformaldehyde (Sigma-Aldrich, St. Louis, MO, USA) for $10 \mathrm{~min}$ at room temperature before being deposited on polylysine-coated slides. After $30 \mathrm{~min}$, the adherent cells were permeabilized by incubation with $0.1 \%$ Triton $\mathrm{X}-100$ (Sigma-Aldrich) for $5 \mathrm{~min}$ at $4^{\circ} \mathrm{C}$. The cells were then incubated with Hoechst 33258 for $30 \mathrm{~min}$ at room temperature, rinsed with PBS and mounted on coverslips using glycerol (Sigma-Aldrich). Finally, cells were visualized using an Olympus BH-2 fluorescence microscope (Olympus, Tokyo, Japan).

Western blot analysis. All of the HL-60 cells treated with AA at various concentrations for $24 \mathrm{~h}$ were collected. The cells were washed twice with PBS and completely lysed in a lysis buffer containing protease inhibitors (Pierce, Thermo Scientific, Waltham, MA, USA). The extracts were centrifuged at $12,000 \mathrm{xg}$ for $15 \mathrm{~min}$ at $4^{\circ} \mathrm{C}$, and the clear supernatants containing the total protein were isolated. The protein concentration was quantified using the BCA assay (Pierce, Thermo Scientific). Equal amounts of protein $(40 \mu \mathrm{g}$ per lane) were separated by 10-12\% SDS-polyacrylamide gel electrophoresis, and the proteins were then transferred to PVDF membranes. The membranes were blocked in $5 \%$ non-fat milk (Sigma-Aldrich) for $2 \mathrm{~h}$ at room temperature and then probed with the specific primary antibodies (1:500) at $4^{\circ} \mathrm{C}$ overnight, and corresponding secondary antibody $(1: 1,000)$ at room temperature for $1 \mathrm{~h}$. The specific protein bands were visualized using an Enhanced Chemiluminescence Detection system (GE Healthcare Bio-Sciences, Pittsburgh, PA, USA).

Statistical analysis. Each experiment was repeated three times. Values are expressed as the mean \pm standard deviation and analyzed using SPSS 13.0 statistical software for Windows (SPSS, Inc., Chicago, IL, USA). The comparisons between each group were analyzed using a one-way analysis of variance and the Student-Newman-Keuls (SNK) test. $\mathrm{P}<0.05$ was considered to indicate a statistically significant difference between values.

\section{Results}

AA inhibits the proliferation of HL-60 cells. An MTT assay was used to detect the cytotoxicity of different concentrations of AA $(0,10,20,30,40,50$ and $60 \mu \mathrm{mol} / \mathrm{l})$ on HL-60 cells for 24 and $48 \mathrm{~h}$ and PBMCs for $24 \mathrm{~h}$. As shown in Fig. 1, the growth of HL-60 cells was found to be inhibited by AA in a time- and dose-dependent manner. The proliferation inhibition rate of HL-60 cells significantly increased following incubation with AA at various concentrations for $24 \mathrm{~h}(\mathrm{P}<0.05)$, while AA showed low toxicity to PBMCs. When the same concentration of AA was used, the proliferative rate of HL-60 cells was shown to be inhibited in a time-dependent manner $(\mathrm{P}<0.05)$. The $\mathrm{IC}_{50}$-values of AA for HL-60 cells at 24 and $48 \mathrm{~h}$ were $46.67 \mu \mathrm{mol} / 1$ and $36.42 \mu \mathrm{mol} / 1$, respectively.

AA induces apoptosis in HL-60 cells. Annexin V-FITC/PI double staining and flow cytometric analysis were utilized to assess the apoptotic rate of HL-60 cells treated with AA, as shown in Fig. 2. The early apoptotic rates of HL-60 cells treated with $0,20,40$ and $60 \mu \mathrm{mol} / 1 \mathrm{AA}$ for $24 \mathrm{~h}$ were $4.58 \pm 2.01,10.45 \pm 2.88$ and $25.68 \pm 5.67 \%$, respectively, which were significantly higher than that of the control group $(2.53 \pm 2.06 \%)$. The late apoptotic rates were $11.20 \pm 2.91$, $20.67 \pm 4.58$ and $60.63 \pm 6.65 \%$, respectively, while that of the control group was significantly lower $(4.33 \pm 1.86 \%)$. To further identify whether AA-induced apoptosis in HL-60 cells is 

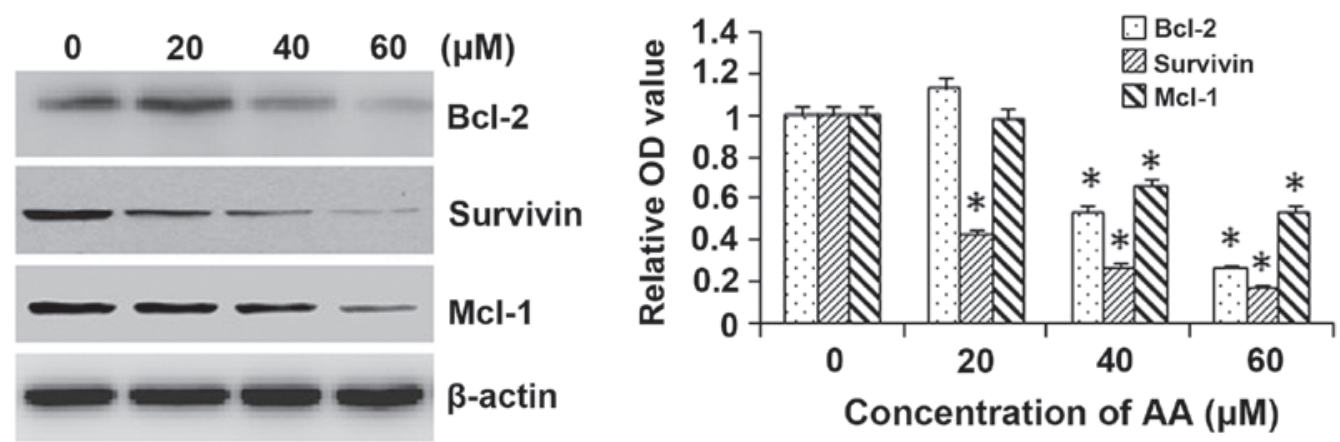

Figure 4. Effect of AA on apoptosis-associated proteins in HL-60 cells. HL-60 cells were treated with 0 , 20, 40 or $60 \mu$ mol/1 AA for 24 h. Cell lysates were prepared and subjected to western blot analysis of Bcl-2, Mcl-1 and survivin. Blots were quantified by densitometric analysis. Values represent the mean \pm standard deviation of three determinations. ${ }^{*} \mathrm{P}<0.05$, as compared with the control cells. AA, asiatic acid; Bcl-2, B-cell lymphoma 2; Mcl, myeloid cell leukemia; OD, optical density.
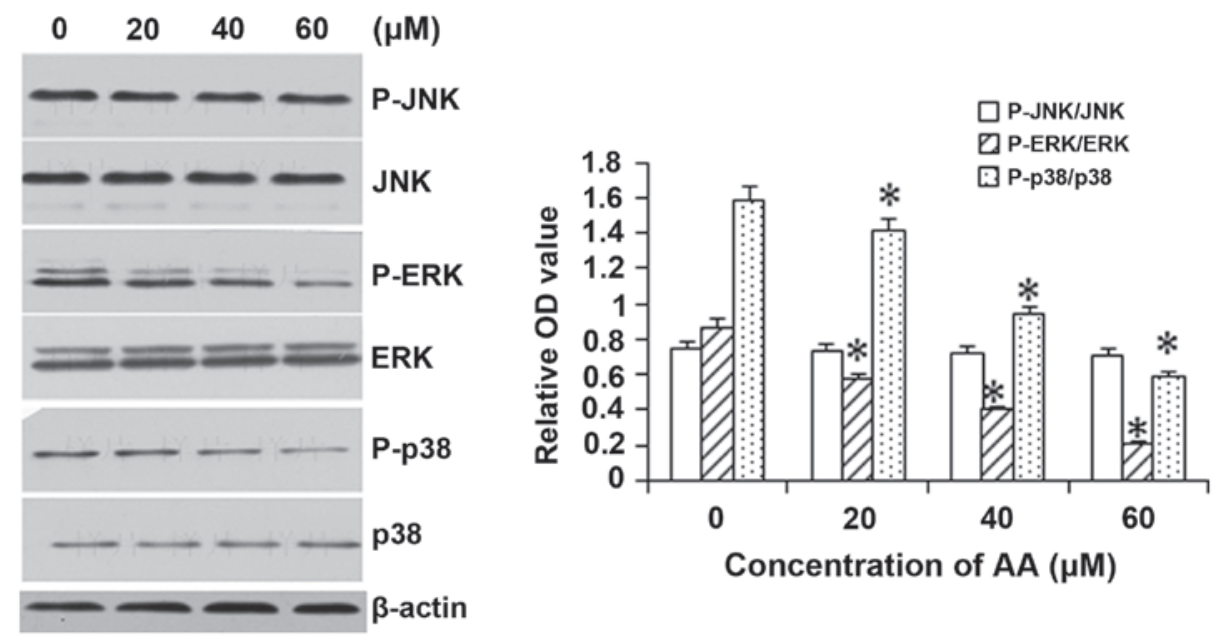

Figure 5. Effect of AA on expression and activation of proteins involved in the MAPK signaling pathway. Cells were treated with $0,20,40$ or $60 \mu$ mol/1 AA for $24 \mathrm{~h}$. The cell lysates were prepared, and western blot was performed using antibodies against total or phosphorylated MAPK-signaling proteins. Blots were quantified by densitometric analysis. Values represent the means \pm standard deviation of three determinations. "P $<0.05$, as compared with the control cells. AA, asiatic acid; MAPK, mitogen-activated protein kinase; p-JNK, phosphorylated c-Jun N-terminal kinase; ERK, extracellular signal-regulated kinase; OD, optical density.

caspase-dependent, caspase inhibitor of Z-VAD-FMK was used in combination with $60 \mu \mathrm{mol} / 1$ AA. Z-DEVD-FMK was found to significantly decrease the apoptotic rate of AA-treated HL-60 cells $(15.72 \pm 3.82 \%, \mathrm{P}<0.05)$.

HL-60 cells treated with AA for $24 \mathrm{~h}$ were also stained with Hoechst 33258 to visualize the nuclear changes. HL-60 cell nuclei were regular in shape in the control group, whereas apparent apoptotic bodies were observed among HL-60 cells after treatment with $40 \mu \mathrm{mol} / 1 \mathrm{AA}$. The nuclei of these apoptotic cells were condensed, and the nuclear envelopes appeared lytic (Fig. 3).

AA decreases the expression of apoptosis-associated proteins in HL-60 cells. To investigate the mitochondrial apoptotic events involved in AA-induced apoptosis, changes in the levels of anti-apoptotic proteins Bcl-2, survivin and $\mathrm{Mcl}-1$ were assessed by western blot analysis. AA treatment attenuated the expression levels of the anti-apoptotic proteins Bcl-2, survivin and Mcl-1 in a concentration-dependent manner in HL-60 cells (Fig. 4). These results suggested that Bcl-2, survivin and Mcl-1 have essential roles in AA-mediated induction of apoptosis.
AA inhibits the activation of ERK and p38 but not JNK. To assess the involvement of the MAPK pathway in the mechanism underlying the cytotoxicity of AA, the levels and activation (phosphorylation) of JNK, p38 and ERK1/2 were assessed in AA-treated HL-60 cells. As shown in Fig. 5, exposure of HL-60 cells to 20,40 and $60 \mu \mathrm{M}$ AA resulted in a significant inhibition of phosphorylation of p38 and ERK1/2 in a dose-dependant manner, while basal activation of p38 and ERK was not altered. However, AA treatment did not JNK levels (including the phosphorylated and unphosphorylated forms).

\section{Discussion}

In previous studies, numerous natural products, including pentacyclic triterpenoids, have been discovered to be potent anti-leukemic agents for AML therapy (5-7). The present study was the first, to the best of our knowledge, to report the cytotoxic activity of AA on the human acute leukemia cell line HL-60. HL-60 cells treated with AA underwent apoptosis in a dose- and time-dependent manner. It was demonstrated that $\mathrm{AA}$ attenuated the expression levels of $\mathrm{Bcl}-2$, survivin 
and Mcl-1, and also the phosphorylation of ERK and p38 in a time-dependent manner.

The Bcl-2 family of proteins consists of pro-apoptotic effector proteins, including $\mathrm{Bcl}-2$ associated $\mathrm{X}$ protein and Bcl-2 homologous antagonist killer, as well as anti-apoptotic proteins, including Bcl-2, Bcl extra large (Bcl-xL) and Mcl-1. Bcl-2 proteins exert anti-apoptotic effects through regulating the permeabilization of the mitochondrial outer membrane, a key step in apoptosis. Their complex network of interactions in the cytosol and mitochondria determines the fate of the cells (17). Cancer cells often violate key cellular checkpoints that would normally drive the cells to die by programmed cell death. As a result, they require to overcome the apoptotic stress either by reducing the expression of pro-apoptotic factors or, more frequently, by upregulating anti-apoptotic molecules, including Bcl-2, BcL-XL and MCL-1 (18,19). Bcl-2 and MCL-1 are critical for the development and maintenance of hematologic malignancies $(20,21)$. Constitutively high levels of Bcl-2 and Mcl-1 have been associated with a more aggressive malignant phenotype and/or drug resistance to various classes of chemotherapeutic agents in cancer $(22,23)$. This anti-apoptotic subfamily of proteins is currently considered a major target in the development of novel methods to improve treatment outcomes for leukemia patients. In the previous decade, several drugs directed at inhibiting Bcl-2 have been tested in the clinic, with several of them showing promising effects, particularly in lymphoid malignancies (24). Retroviruses encoding BimSL62A/F69A, which selectively bind Mcl-1, were able to selectively diminish survival of cells in two samples of clinical AML (21). In the present study, AA was demonstrated to decrease the anti-apoptotic activity in HL-60 cells through downregulating Bcl-2 and Mcl-1 expression, in consistency with the previously reported effect of AA on Bcl-2 expression in colon cancer, melanoma and breast cancer cells $(15,25,26)$.

Survivin (BIRC5) is a member of the family of inhibitors of apoptosis proteins (IAPs) and has been implicated in the control of cell survival as well as regulation of mitosis in cancer, including solid tumors and hematological malignancies (27-29). Upon activation of pro-apoptotic cell signaling, survivin is released from the mitochondria and inhibits caspases-3 and -9 . This function requires association with hepatitis B X-interacting protein and/or with X-linked IAP and is inhibited by SMAC-DIABLO $(27,28)$. The regulation of survivin expression and function is complex and occurs at various levels, including transcription, differential splicing, protein degradation and intracellular sequestration via different ligands (28). Overexpression of suvivin is correlated with advanced disease, accelerated time of recurrence, reduced survival and resistance to therapy (30). AML patients with overexpression of survivin showed an unfavorable response to chemotherapy in $81.2 \%$ of the patients and shorter median survival time (30 days) compared to that of patients with normal expression (29). Thus, targeting survivin with small-molecule inhibitors by their anti-sense approaches or natural IAP antagonist mimetics may be an attractive strategy of anti-leukemia treatment. Such agents can either directly induce apoptosis of tumor cells or sensitize them to other cytotoxic agents, hence overcoming drug resistance $(31,32)$. Thereofore, the present study evaluated the effect of AA on survivin in HL-60 cells. The results showed that, in addition to bcl-2 protein, survivin is likely to be involved in the mechanism of AA-induced apoptosis.

MAPKs, a family of serine/threonine kinases, are mediators of intracellular signals in response to various stimuli. JNK, p38 and ERK1/2 are the three main members of three different MAPK pathways that can be activated by growth factors, DNA damage, cytokines, oxidant stresses, UV light, anti-cancer drugs and osmotic shock $(33,34)$. These signaling pathways regulate a variety of cellular activities, including proliferation, differentiation, survival and death. Deviation from the strict control of MAPK signaling pathways has been implicated in the development of numerous human diseases, including Alzheimer's disease (AD), Parkinson's disease (PD), amyotrophic lateral sclerosis (ALS) and various types of cancer (35). The present study showed that AA significantly inhibited phosphorylation of p38 and ERK without affecting the JNK pathway. According to previous studies, the role of these three MAPK pathways in cancer has remained controversial, as MAPK pathways have been shown to mediate pro-apoptotic as well as anti-apoptotic signals in different systems, apparently depending on the stimulus and cell type involved. For instance, the apoptosis of K562 cells induced by icaritin was accompanied by the inhibition of activation of p-ERK and p-P38, while activation of p-ERK and p-P38 were shown to be critical mediators in AA-induced cell growth inhibition of human breast cancer cell lines $(26,36)$. JNK and p38 are activated by cellular stress and have been associated with apoptosis (37). However, certain studies have indicated that the JNK is required for interleukin-3-mediated cell survival and that $\mathrm{p} 38$ is associated with the development of chemoresistance by activating nuclear factor kappa B $(38,39)$.

In conclusion, the results of the present study suggested that AA induced apoptosis through inhibiting Bcl-2, survivin and MAPK signaling pathways in HL-60 human acute leukemia cells. These results strongly suggested that AA may be a valuable agent for molecular targeted therapy of human acute leukemia.

\section{Acknowledgements}

The authors would like to acknowledge Dr Fei Zhao (Institute of Hematology, Union Hospital, Tongji Medical College, Huazhong University of Science and Technology, Wuhan, China) for analyzing the data.

\section{References}

1. Ben-Ayre E, Attias S, Tadmor T and Schiff E: Herbs in hemato-oncological care: An evidence-based review of data on efficacy, safety, and drug interactions. Leuk Lymphoma 51: 1414-1423, 2010.

2. Wang N, Li DY, Niu HY, et al: 2-hydroxy-3-methylanthraquinone from Hedyotis diffusa Willd induces apoptosis in human leukemic U937 cells through modulation of MAPK pathways. Arch Pharm Res 36: 752-758, 2013.

3. Li Q, Huai L, Zhang C, et al: Icaritin induces AML cell apoptosis via the MAPK/ERK and PI3K/AKT signal pathways. Int J Hematol 97: 617-623, 2013

4. Yu Z, Wang R, Xu L, Xie S, Dong J and Jing Y: $\beta$-Elemene piperazine derivatives induce apoptosis in human leukemia cells through downregulation of c-FLIP and generation of ROS. PLoS One 6: e15843, 2011.

5. Shin SW and Park JW: Ursolic acid sensitizes prostate cancer cells to TRAIL-mediated apoptosis. Biochim Biophys Acta 1833: 723-730, 2013. 
6. Liu L, Fu J, Li T, et al: NG, a novel PABA/NO-based oleanolic acid derivative, induces human hepatoma cell apoptosis via a ROS/MAPK-dependent mitochondrial pathway. Eur J Pharmacol 691: 61-68, 2012.

7. Wang P, Li Q, Li K, et al: Betulinic acid exerts immunoregulation and anti-tumor effect on cervical carcinoma (U14) tumor-bearing mice. Pharmazie 67: 733-739, 2012.

8. Tang LX, He RH, Yang G, et al: Asiatic acid inhibits liver fibrosis by blocking TGF-beta/Smad signaling in vivo and in vitro. PLoS One 7: e31350, 2012.

9. Liu J,He T, Lu Q, Shang J, Sun H and Zhang L: Asiatic acid preserves beta cell mass and mitigates hyperglycemia in streptozocin-induced diabetic rats. Diabetes Metab Res Rev 26: 448-454, 2010.

10. Lee KY, Bae ON, Serfozo K, et al: Asiatic acid attenuates infarct volume, mitochondrial dysfunction and matrix metalloproteinase- 9 induction after focal cerebral ischemia. Stroke 43: 1632-1638, 2012.

11. Somboonwong J, Kankaisre M, Tantisira B and Tantisira MH: Wound healing activities of different extracts of Centella asiatica in incision and burn wound models: an experimental animal study. BMC Complement Altern Med 12: 103, 2012.

12. Kavitha CV, Agarwal C, Agarwal R and Deep G: Asiatic acid inhibits pro-angiogenic effects of VEGF and human gliomas in endothelial cell culture models. PLoS One 6: e22745, 2011.

13. Lee YS, Jin DQ, Kwon EJ, et al: Asiatic acid, a triterpene, induces apoptosis through intracellular $\mathrm{Ca} 2+$ release and enhanced expression of p53 in HepG2 human hepatoma cells. Cancer Lett 186: 83-91, 2002.

14. Tang XL, Yang XY, Jung HJ, et al: Asiatic acid induces colon cancer cell growth inhibition and apoptosis through mitochondrial death cascade. Biol Pharm Bull 32: 1399-1405, 2009.

15. Park BC, Bosire KO, Lee ES, Lee YS and Kim JA: Asiatic acid induces apoptosis in SK-MEL-2 human melanoma cells. Cancer Lett 218: 81-90, 2005.

16. Yoshida M, Fuchigami M, Nagao T, et al: Antiproliferative constituents from Umbelliferae plants VII. Active triterpenes and rosmarinic acid from Centella asiatica. Biol Pharm Bull 28: $173-175,2005$

17. Reed JC: Bcl-2-family proteins and hematologic malignancies: history and future prospects. Blood 111: 3322-3330, 2008

18. Hanahan D and Weinberg RA: Hallmarks of cancer: the next generation. Cell 144: 646-674, 2011.

19. Ni Chonghaile T, Sarosiek KA, Vo TT, et al: Pretreatment mitochondrial priming correlates with clinical response to cytotoxic chemotherapy. Science 334: 1129-1133, 2011.

20. Reed JC and Pellecchia M: Apoptosis-based therapies for hematologic malignancies. Blood 106: 408-418, 2005.

21. Glaser SP, Lee EF, Trounson E, et al: Anti-apoptotic Mcl-1 is essential for the development and sustained growth of acute myeloid leukemia. Genes Dev 26: 120-125, 2012.

22. Perego P, Righetti SC, Supino R, et al: Role of apoptosis and apoptosis-related proteins in the cisplatin-resistant phenotype of human tumor cell lines. Apoptosis 2: 540-548, 1997.

23. Campbell KJ, Bath ML, Turner ML, et al: Elevated Mcl-1 perturbs lymphopoiesis, promotes transformation of hematopoietic stem/progenitor cells and enhances drug resistance. Blood 116: 3197-3207, 2010.
24. Robinson BW, Behling KC, Gupta M, et al: Abundant anti-apoptotic BCL-2 is a molecular target in leukaemias with $\mathrm{t}$ (4;11) translocation. Br J Haematol 141: 827-839, 2008.

25. Bunpo P, Kataoka K, Arimochi H, et al: Inhibitory effects of asiatic acid and CPT-11 on growth of HT-29 cells. J Med Invest 52: 65-73, 2005

26. Hsu YL, Kuo PL, Lin LT and Lin CC: Asiatic acid, a triterpene, induces apoptosis and cell cycle arrest through activation of extracellular signal-regulated kinase and p38 mitogen-activated protein kinase pathways in human breast cancer cells. J Pharmacol Exp Ther 313: 333-344, 2005.

27. Tamm I, Wang Y, Sausville E, et al: IAP-family protein survivin inhibits caspase activity and apoptosis induced by Fas (CD95), Bax, caspases and anticancer drugs. Cancer Res 58: 5315-5320, 1998.

28. Mita AC, Mita MM, Nawrocki ST and Giles FJ: Survivin: key regulator of mitosis and apoptosis and novel target for cancer therapeutics. Clin Cancer Res 14: 5000-5005, 2008.

29. Ibrahim AM, Mansour IM, Wilson MM, Mokhtar DA, Helal AM and Al Wakeel HM: Study of survivin and X-linked inhibitor of apoptosis protein (XIAP) genes in acute myeloid leukemia (AML). Lab Hematol 18: 1-10, 2012.

30. Adida C, Berrebi D, Peuchmaur M, Reyes-Mugica M and Altieri DC: Anti-apoptosis gene, survivin and prognosis of neuroblastoma. Lancet 351: 882-883, 1998.

31. Smolewski P and Robak T: Inhibitors of apoptosis proteins (IAPs) as potential molecular targets for therapy of hematological malignancies. Curr Mol Med 11: 633-649, 2011.

32. Zhu Z, Li E, Liu Y, et al: Bufalin induces the apoptosis of acute promyelocytic leukemia cells via the downregulation of survivin expression. Acta Haematol 128: 144-150, 2012.

33. Johnson GL and Lapadat R: Mitogen-activated protein kinase pathways mediated by ERK, JNK and p38 protein kinases. Science 298: 1911-1912, 2002.

34. Olson JM and Hallahan AR: p38 MAP kinase: a convergence point in cancer therapy. Trends Mol Med 10: 125-129, 2004.

35. Kim EK and Choi EJ: Pathological roles of MAPK signaling pathways in human diseases. Biochim Biophys Acta 1802: 396-405, 2010

36. Zhu JF, Li ZJ, Zhang GS, et al: Icaritin shows potent anti-leukemia activity on chronic myeloid leukemia in vitro and in vivo by regulating MAPK/ERK/JNK and JAK2/STAT3 /AKT signalings. PLoS One 6: e23720, 2011

37. Huh JE, Kang KS, Chae C, Kim HM, Ahn KS and Kim SH: Roles of p38 and JNK mitogen-activated protein kinase pathways during cantharidin-induced apoptosis in U937 cells. Biochem Pharmacol 67: 1811-1818, 2004.

38. Hendrickx N, Volanti C, Moens U, et al: Up-regulation of cyclooxygenase- 2 and apoptosis resistance by p38 MAPK in hypericin-mediated photodynamic therapy of human cancer cells. J Biol Chem 278: 52231-52239, 2003.

39. Yu C, Minemoto Y, Zhang J, et al: JNK suppresses apoptosis via phosphorylation of the proapoptotic $\mathrm{Bcl}-2$ family protein BAD. Mol Cell 13: 329-340, 2004. 